\title{
SURFACE PLASMON RESONANCE IMAGING: application in microbiology
}

Marine BOULADE ${ }^{1}$, Julien MOREAU ${ }^{2}$, Thierry LIVACHE $^{1}$

${ }^{1}$ CEA, CNRS, Univ. Grenoble Alpes, INAC-SPRAM, 38000 Grenoble, France

${ }^{2}$ Laboratoire Charles Fabry, Institut d'Optique Graduate School,

Université Paris Saclay, 91227 Palaiseau, France

julien.moreau@institutoptique.fr

Surface plasmons can be defined as the collective oscillation of free electrons on a metallic surface. They were first observed back in the early 20th century, by the American physicist and inventor R.W. Wood, while he was studying the diffraction of light by a metallic grating. Wood observed very narrow absorption bands in the diffraction spectrum that would remain unexplained until 1941, when U. Fano demonstrated that they were associated with surface waves known today as surface plasmons. A similar phenomenon explains the colours of stained-glass windows in cathedrals, as the glass contains metallic nanoparticles with plasmon absorption bands in the visible spectrum.

Surface plasmon resonance (SPR) is induced by incident photons. This resonant coupling can only take place if several conditions are met [1]. First, the real part of the metal's permittivity at the excitation wavelength must be negative. This is the case for gold, silver and aluminium in the visible and near-infrared spectrum. Second, the wave vector of the incident light must match that of the surface plasmon:

$$
\frac{2 \pi}{\lambda} n_{i n c} \sin \theta=\frac{2 \pi}{\lambda} \operatorname{Re}\left\{\sqrt{\frac{\varepsilon_{m} \varepsilon_{d}}{\varepsilon_{m}+\varepsilon_{d}}}\right\}
$$

where $\theta$ is the angle of incidence, $\lambda$ the incident wavelength, $n_{i n c}$ the refractive index of the incident medium (prism in Fig. 1), and $\varepsilon_{m}$ and $\varepsilon_{d}$ the permittivities of the metal and the dielectric (water in Fig. 1). Surface plasmons are actually just one (classic) example of waves guided by total reflection, but at the interface between a dielectric and a metal sheet.

The coupling condition is usually achieved with an optical configuration developed by E. Kretschmann and H. Raether in 1968 [2], in which transverse magnetic (TM)-polarized incident light passes through a prism coated with a metal film (Fig. 1). The coupling between the photons and the metal's electrons manifests itself in a sharp drop in reflectivity for a particular angle of incidence, determined by the incident wavelength.

\section{small components MASSIVE IMPACT}

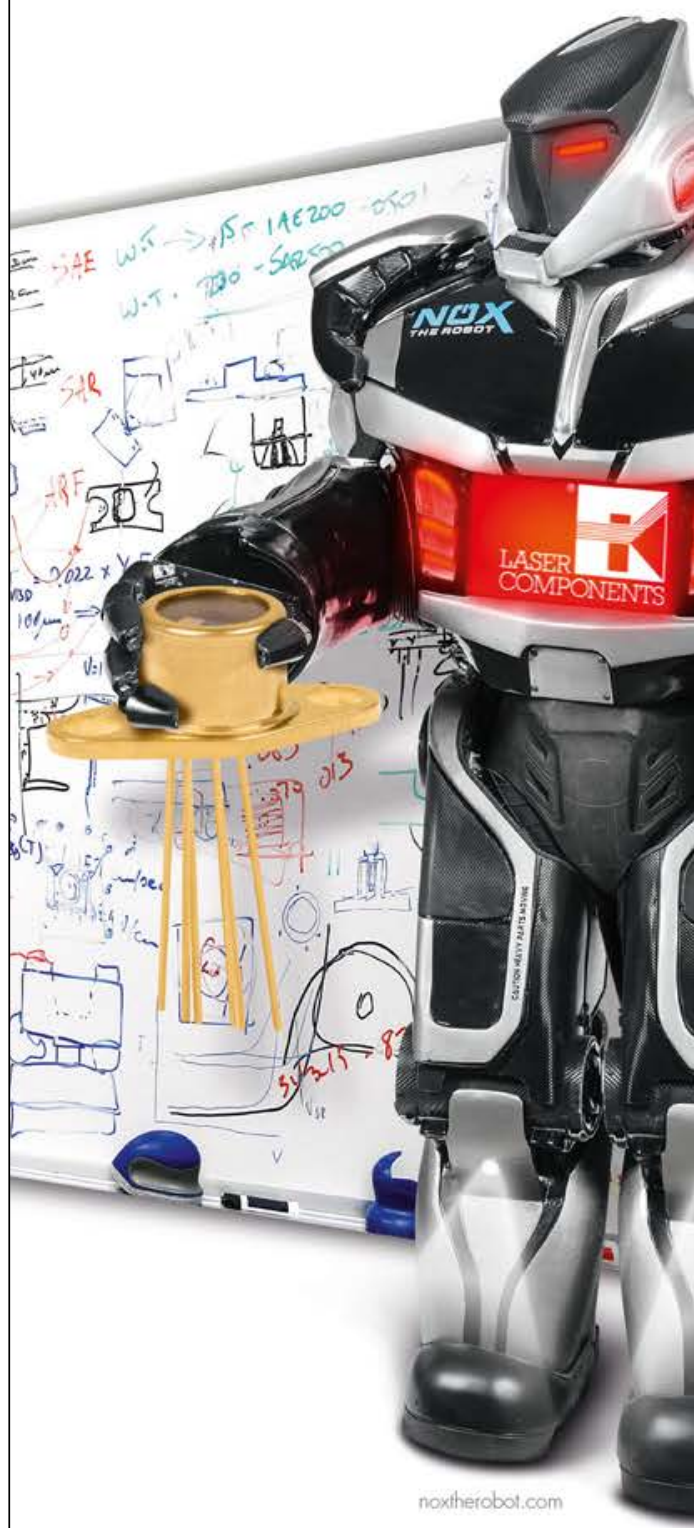

We Manufacture IR Detectors

- (x) InGaAs Photodiodes

- PbS \& PbSe Detectors

- Pyroelectric Detectors 


\section{$\mathrm{FICHOU}$ CAPABILITIES}

\author{
YOUR PARTNER TO MEET \\ YOUR UNIQUE NEED. \\ FICHOU OFFERS THE IDEAL \\ KNOWLEDGE OF CUSTOM OPTIC \\ MANUFACTURE ASSOCIATED WITH \\ CUSTOM OPTICAL COATING \\ DESIGN TO MEET YOUR NEEDS.
}

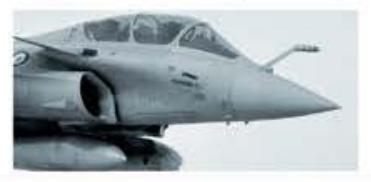

PRODUCTS

\section{- CUBE, PRISM, MIRROR}

- WAVEPLATE, POLARIZER

- LENS, BEAMSPLITTER,

- WINDOW, ...

- CRYSTAL, ROD, ...

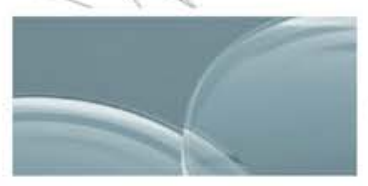

\section{COATINGS}

- ANTIREFLECTION COATING

- DIELECTRIC COATING

- metallic coatinc

- INFRARED coATING

\section{- CUSTOMIZED COATING}

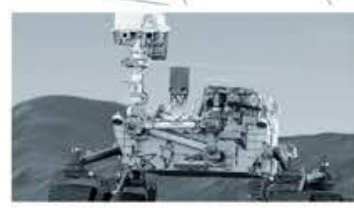

\section{CUSTOM REALIZATIONS}

- pyRAmid

- large sapphire window

- polarization optics

- polarization sCRAMBLER

- WAVEPLATE 150 mM diameter

- achromatic RETARDATION PLATE

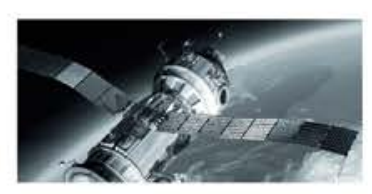

Certificated by AECMA EASE in accordance of EN9100 WWW. Optique-fichou.com Fichou : 30 , Rue de la Garenne, 94260 Fresnes, FRANCE
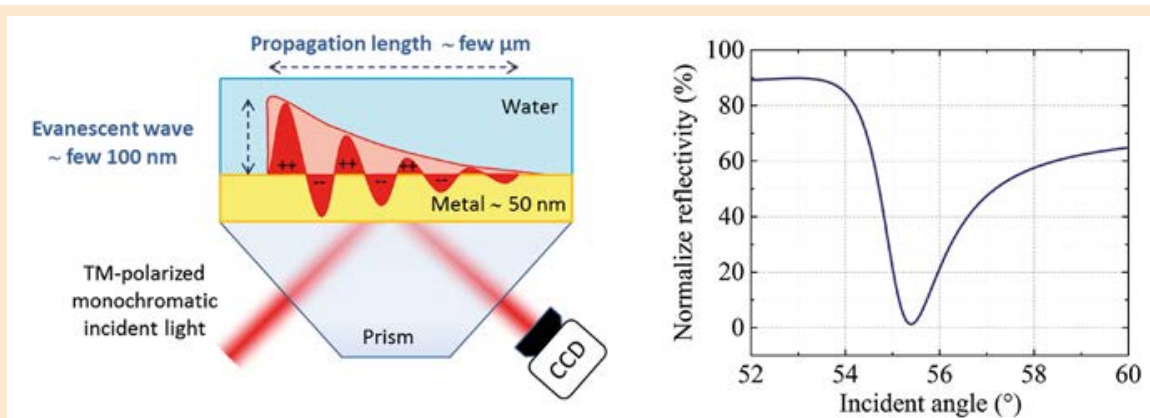

Figure 1. Diagram illustrating the principle of an SPR system: a quasi-monochromatic, collimated TM-polarized light illuminates a metallic film deposited on a prism. An evanescent, plasmon-type wave is generated at the metal-water interface, resulting in an absorption resonance within an ultra-narrow angle (less than $1^{\circ}$ ).

The excited surface plasmon propagates along the metal-dielectric interface. This evanescent wave typically has a penetration depth of several hundred nanometres and a propagation length of several microns. It was the sharpness of the resonance and the evanescence of the surface plasmons that led to the use of SPR in sensors, which first appeared on the market in the early 1980s.

\section{Principle of SPR imaging}

Most commercial and research SPR systems work in exactly the same way. A collimated, quasi-monochromatic beam, generally in the red spectrum for greater sensitivity, is directed through a prism onto a $50 \mathrm{~nm}$-thick gold film deposited either on a glass slide or else on the prism itself. The detection principle relies directly on the resonance (dependent on the permittivity or refractive index of the dielectric above the gold film) and evanescent quality of the surface plasmons. In this coupling condition, the optical device becomes a transducer that converts local variations in the optical index in the vicinity of the metal to large optical signals, as a result of changes in the coupling efficiency between incident light and plasmons. Insofar as these variations in the index are proportional to mass density, SPR sensors can be regarded as optical microbalances, accurate to within a few picogrammes per $\mathrm{mm}^{2}$.

Numerous detection configurations have been devised to measure these resonance shifts. The one with the simplest instrumentation - and also the most widely used - consists in measuring variations in incident beam reflectivity, preferably at the steepest slope in the plasmon resonance. A camera is used to track these variations in reflectivity across the entire gold surface, resulting in plasmonic imaging (SPRi). A microfluidic system is generally placed on the metal surface, so that the substances to be analysed can be sequentially injected. For example, the adsorption of a single layer of organic molecules

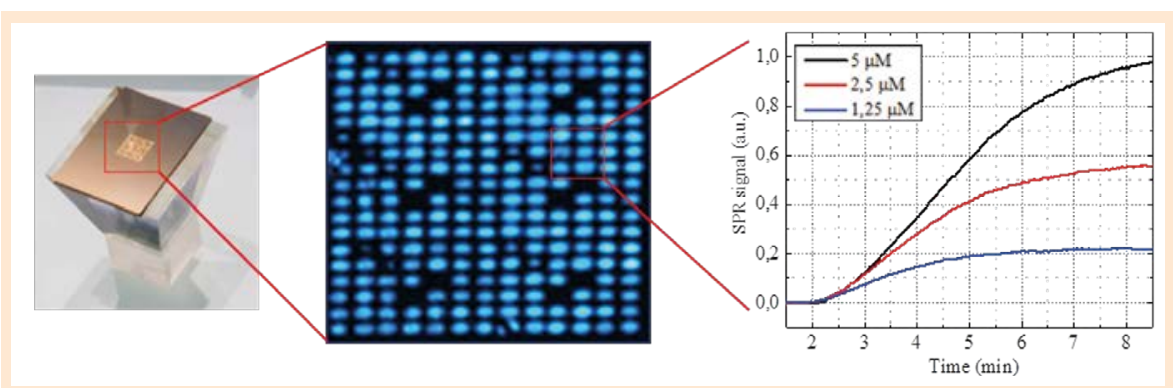

Figure 2. Photo of a functionalized gold film, showing the microarray of probes on the chip. Image of reflectivity variation in SPRi (pseudo-coloured) after a DNA-DNA interaction. Example of kinetics measured on three families of probes with different surface concentrations. 
of nanometric thickness typically brings about a variation in incident beam reflectivity of nearly $2 \%$. The same molecular adsorption on a plain glass slide with no gold film would yield a variation of just $0.00002 \%$. The metal film and the surface plasmons therefore amplify sensitivity fivefold.

As with other biosensors, biochips derive their specificity from their surface functionalization. The main strength of SPRi sensors is that they do not need any secondary marker on the target molecule (so-called label-free technique). Rather, it is the variation in the optical index close to the gold layer, brought about by the arrival of the molecules, that is detected. This avoids all the constraints linked to marking, such as the marker's impact on the interaction under scrutiny, photobleaching for fluorescent markers, and end-point readings. All SPRi systems therefore rely on some form of surface chemistry to enable target-specific molecular probes to be attached to the gold surface. A wide range of surface chemistries have been developed, allowing virtually every major class of biomolecule to be attached, including oligonucleotides, antibodies, proteins and peptides. Between several tens and several hundreds of probes can be deposited on the surface of a single chip, meaning that the interactions between a single target molecule and a large number of probes can be studied simultaneously [3]. The other advantage of SPRi is that the measurements are made in real time, allowing for video-rate imaging of reflectivity across the chip (Fig. 2). This enables probe-target kinetics to be captured, and association and dissociation constants to be determined.

\section{Application in microbiology}

When a very small number of bacteria need to be detected - typically between one and several hundred, the standard methods used in the fields of medicine or food processing for microbiological diagnosis rely on the growth properties of these bacteria. There has to be an incubation period of at least 18 hours before any analyses can be performed, and it generally takes at least 36 hours for a pathogenic agent to be identified [4]. Such lengthy waits are extremely problematic if pathogenic organisms urgently need to be identified. For example, as fresh foodstuffs are highly perishable, they are sometimes put on sale before the results of bacterial tests are known. This obviously represents a health risk for customers, as well as an economic risk for manufacturers, should a contaminated product subsequently have to be withdrawn from supermarket shelves. Similarly, in the medical field, rapid identification of an infectious pathogenic agent allows patients to start on the most appropriate - and therefore most effective - treatment at an earlier stage. There is thus a very real need to introduce new, more efficient tools that can look for and rapidly identify several pathogens at the same time.

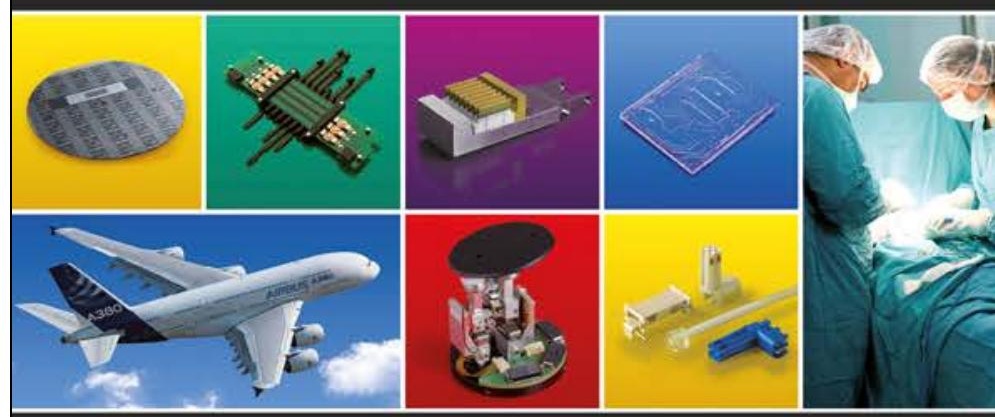

\section{Precision / Miniaturisation Integration of complex functions}

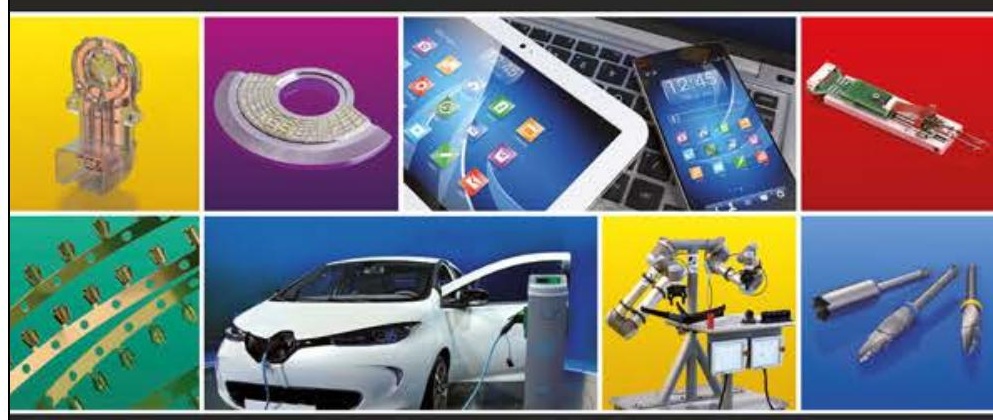

\section{5 - 28 Sept. 2018} Besançon - France

Assembly, Additive manufacturing, Microassembly, Automation, Robotics, Engineering, Interconnection technologies, Laser technology, Overmoulding, Machining, Micromachining, Injection, Metrology, Measurement, Control, Microelectronic packaging, Micromanufacturing, Nanotechnology, Stamping, Fine blanking, Precision bar turning, Surface treatment, Tools ... 


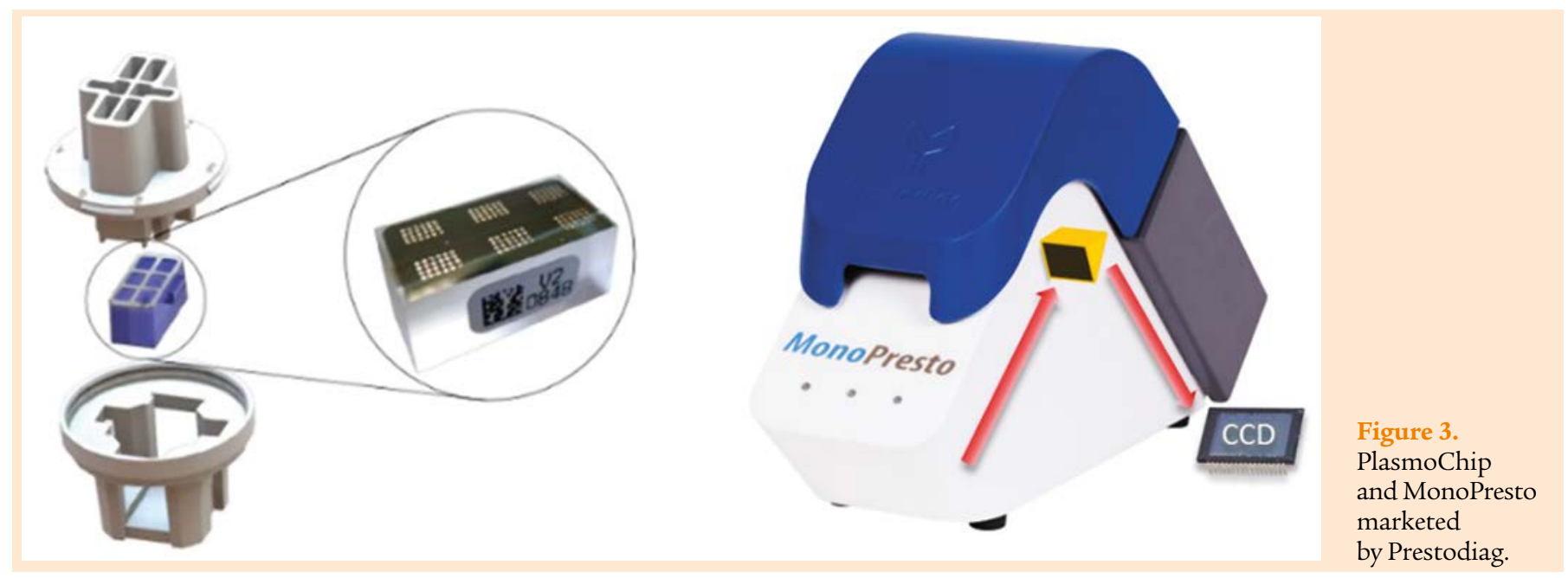

SPR systems offer a simple means of identifying bacteria in real time, without any markers. The bacteria interact with specific ligands grafted on the chip, bringing about a local change in the refractive index in the vicinity of the surface and thus a plasmonic resonance signal [5]. Using SPR systems in imaging mode allows numerous different probes to be attached to the chip's surface, meaning that numerous pathogens can be simultaneously identified in the course of a single test. When proteins and/or antibodies are used as biorecognition molecules, it is possible to detect up to several hundred specific pathogens in the space of just a few hours. This SPR detection method is currently used in bacteriology to detect microbiological pathogens in foodstuffs.
The PlasmoChip biochip (Fig. 3), which can be microarrayed with ligand spots (up to 70 per well) allows for the multiplex detection of pathogenic agents. Depending on the specificity of the ligand that is used, which can range from a peptide to a monoclonal antibody, it is possible to detect bacteria with more or less precision, from a whole genus down to a specific serotype, with a detection threshold of approximately $10^{5}$ bacteria per $\mathrm{mL}$.

Figure 4 illustrates the detection of one particular pathogenic bacterial strain: Cronobacter sakazakii. In just 10 minutes, there is specific recognition of the antibodies and a significant variation in the signal from the $\mathrm{C} 5 \mathrm{a}$ and $\mathrm{C} 5 \mathrm{~b}$ antibody spots specific to this

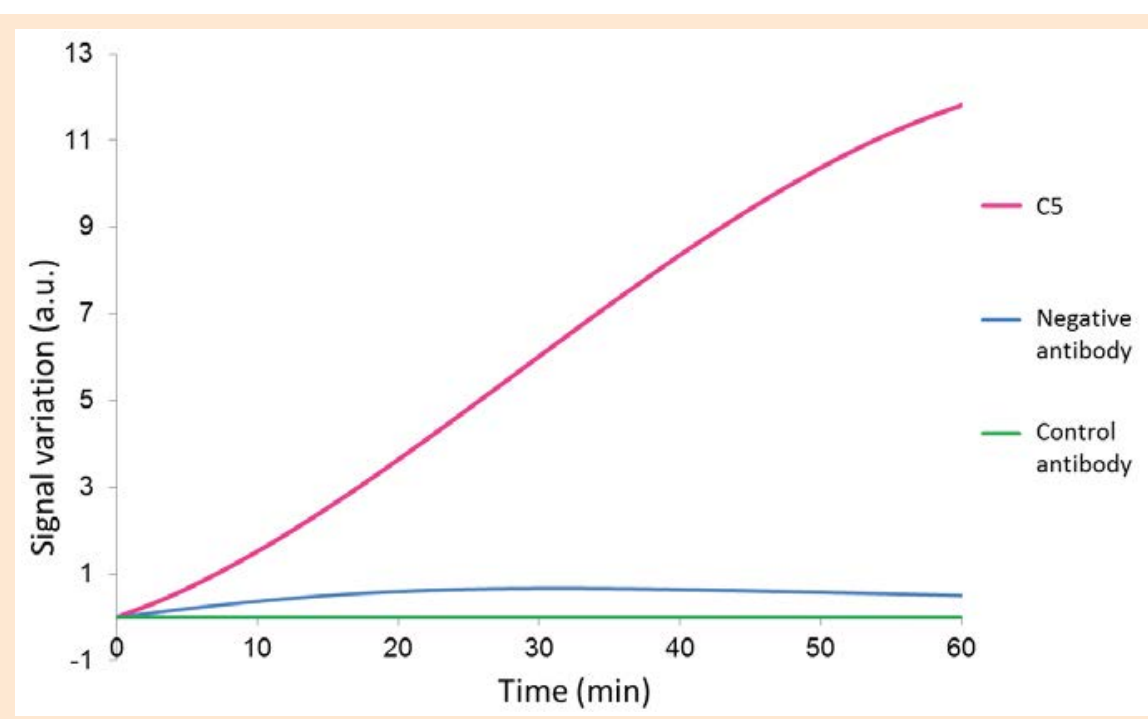

Figure 4. Kinetics of the various ligands measured during the detection of the pathogenic bacterium Cronobacter sakazakii. particular bacterial strain, demonstrating the presence of this bacterium growing in the solution.

The main advantage of using SPR systems to detect bacteria is that analyses can be performed in complex environments. Because they operate in reflection configuration (i.e. opposite side to the medium being analysed), readings are largely impervious to the turbidity, or rather the opacity or absorbance, of the environment. Lastly, as the plasmon field is only sensitive to a depth of a few hundred nanometres, it is relatively unaffected by the potential presence of bulkier compounds (food fragments). This is a considerable advantage over other spectroscopic or colorimetric techniques which, by their very nature, can only work in clean environments. It is this compatibility with complex environments that makes SPR systems so suitable for use in the food processing field, where tests often need to be carried out rapidly on opaque beverages (e.g., milk) or cloudy mixtures where foodstuffs have been put in suspension.

When a foodstuff is contaminated by a pathogen, the latter's concentration is far below the detection threshold of the analytical systems that are currently on the market and, as we saw earlier, a bacterial culture therefore has to be performed, in order to increase the concentrations of the different populations. Using SPR systems in the culture/capture/measure mode can take several hours, but is still 


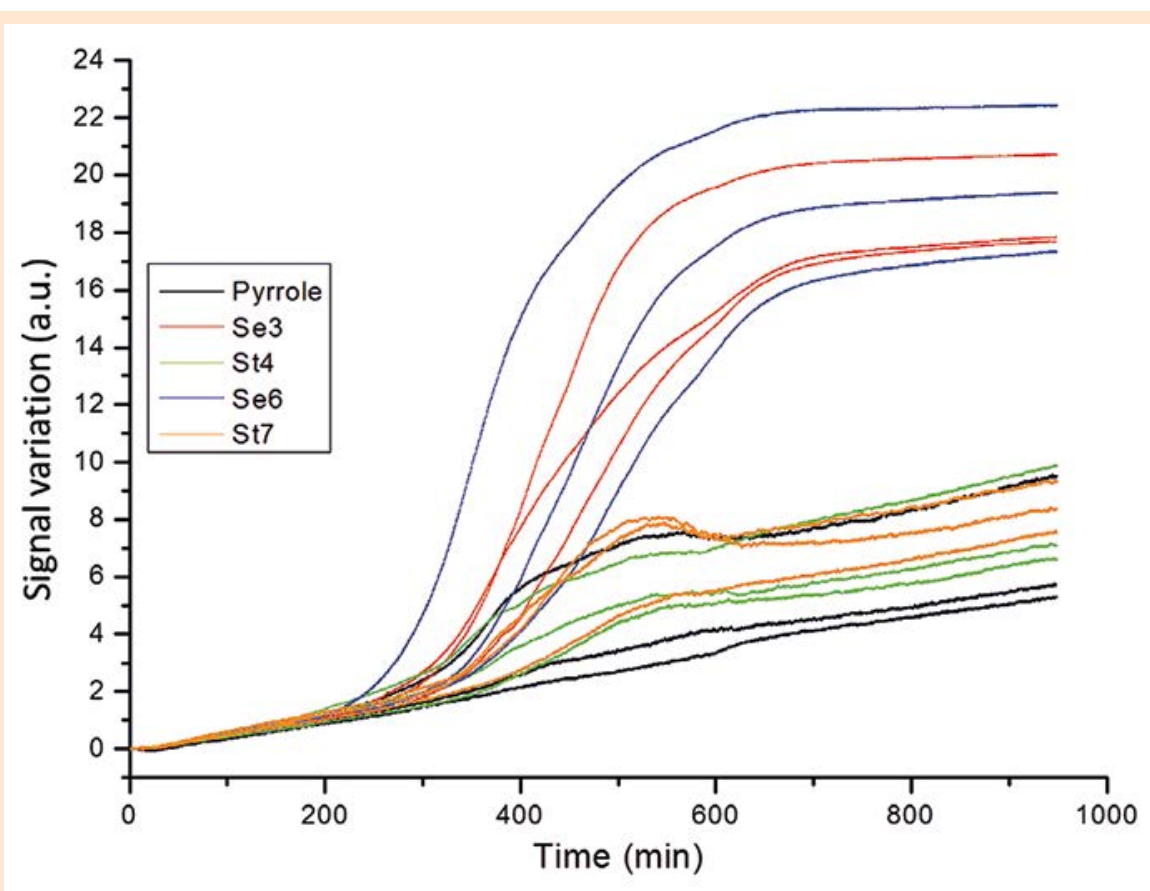

Figure 5. Detection of 10 Salmonella Enteritidis bacteria in raw cow's milk.

faster than standard techniques [6]. Figure 5 illustrates the analysis of raw cow's milk contaminated with ten or so Salmonella Enteritidis bacteria. This particular raw milk possessed a highly developed natural bacterial flora, in the order of $10^{6}$ bacteria per $\mathrm{mL}$. The aim was therefore to detect solely the pathogenic strain(s), from among the many nontarget bacteria. Only Se 3 and Se6 antibodies specific to the $S$. Enteritidis strain exhibited an exponential increase in the SPR signal, reflecting bacterial growth. Some individual pathogenic bacteria can therefore be specifically detected in fewer than 10 hours.
To conclude, SPR and SPRi are proving particularly suitable for carrying out microbiological analyses of complex samples, as these techniques require no marking, take place in real time, and can be multiplexed. Biochip use makes for considerable flexibility and high specificity of the ligand arrays for the target bacteria. Nonetheless, a better understanding of the mechanisms by which bacteria bind to antibodies, and how they multiply close to a surface, through more accurate observations at the single-cell level, could allow for even more targeted, and thus more rapid, assays for pathogenic bacteria.

\section{REFERENCES}

[1] J. Homola, Surface plasmon resonance based sensors, Springer Series in Chemical sensors and biosensors, vol. 4

[2] E. Kretschmann, H. Raether, Radiative decay of nonradiative surface plasmons excited by light, Z. Naturforsch. A 23, 2135 (1968)

[3] L. Leroy, E. Maillart, T. Livache, Biological Applications of Surface Plasmon Resonance Imaging, Springer Series on Chemical Sensors and Biosensors (2012)

[4] V.Velusamy, K. Arshak, O. Korostynska, K. Oliwa, C. Adley, An overview of foodborne pathogen detection: In the perspective of biosensors, Biotechnology advances 28, 232 (2010)

[5] V. Templier, A. Roux, Y. Roupioz, T. Livache, Ligands for label-free detection of whole bacteria on biosensors: A review, Trends in Analytical Chemistry (2015)

[6] S. Bouguelia, Y. Roupioz, S. Slimani, L. Mondani, M.G. Casabona, C. Durmort, T. Livache, On-chip microbial culture for the specific detection of very low levels of bacteria, Lab on a Chip 13 $4024(2013)$

ADVERTORIAL

\section{ELS Embedded Lighting Systems:}

An Advanced Master gathering every competency for a high level training.

Embedded Lighting Systems are undergoing profound changes thanks to new light sources as LED, Laser LEDs or OLEDs, and thanks to new intelligent headlamps as ADB systems, with more and more precision and new functions from Matrix beam to Pixel Lighting. To meet the growing need for resources in this domain worldwide, four major industrial companies PSA group, Renault group, Automotive Lighting and Valeo have asked four higher education institutions, all leaders in their fields. The Institut Optique Graduate School for photonics, ESTACA for transportation systems and Strate School of design for automotive design have thus created an Advanced Master specialized in Embedded Lighting in 2015. This high level training $100 \%$ in English is giving to engineers, already working or recently graduated, an exceptional level of knowledge and experience thanks to the expertise from professors and industry experts. The degree as well as each of the following modules can also be accessed through continuing education:

- Fundamentals of optics

- Photometry

- Systems Engineering

- Mechatronic modelling

- Creative design

- Light sources

- Advanced Photometry

- Environment constraints

- Advanced Mechatronics

- Information systems

- Surface simulations

- Appearance rendering

This chair is also supported by our associated partners Osram, Bertrandt and Mentor Graphics. 